\title{
A neonatal case of HDR syndrome and a vascular ring with a novel GATA3 mutation
}

\author{
Moe Kusakawa $\mathbb{0}^{1,2}$, Takeshi Sato ${ }^{2}$, Ai Hosoda ${ }^{1}$, Eriko Araki ${ }^{1}$, Yohei Matsuzaki ${ }^{1}$, Yukio Yamashita', Jun Ishihara', \\ Yoshinori Inagaki ${ }^{3}$, Noboru Uchida², Tomohiro Ishii $\mathbb{C}^{2}$ and Tomonobu Hasegawa ${ }^{2}$
}

\begin{abstract}
HDR syndrome (OMIM \#146255) is caused by haploinsufficiency of the GATA3 gene. A vascular ring has not been reported in patients with GATA3-associated HDR syndrome. We report a neonatal case of HDR syndrome and a vascular ring that were possibly due to a novel frameshift mutation in the GATA3 gene.
\end{abstract}

HDR syndrome is characterized by the triad of hypoparathyroidism, sensorineural deafness, and renal disease [1]. This disorder is caused by haploinsufficiency of the GATA3 gene related to disruption of the zinc-finger domain of GATA3. A vascular ring, which consists of abnormal blood vessels, is a congenital malformation of the aortic arch and its branches surrounding the trachea and esophagus. To date, a vascular ring has not been reported in patients with GATA3-associated HDR syndrome. We report a case of HDR syndrome with a vascular ring in a patient who had a novel GATA3 mutation.

The proband was the first child of healthy, nonconsanguineous Japanese parents. He was suspected to have a vascular ring by fetal ultrasound evaluation. He was born at full-term, with a birth weight of $2610 \mathrm{~g}(-0.1 \mathrm{SD})$ and length of $50.4 \mathrm{~cm}(+1.6 \mathrm{SD})$. A contrast computed tomography scan and echocardiography showed that the right aortic arch, aberrant origin of the left subclavian artery, and the left arterial duct formed a vascular ring (Fig. 1). On the 24th day of life, he exhibited generalized convulsions due to hypocalcemia $(6.0 \mathrm{mg} / \mathrm{dL}$, reference 9.0-11.0). He also had hyperphosphatemia $(10.8 \mathrm{mg} / \mathrm{dL}$, reference 5.0-7.7) and relatively low levels of intact parathyroid hormone $(38 \mathrm{pg} / \mathrm{mL}$, reference 10-65),

\footnotetext{
Correspondence: Tomohiro Ishii (tishii@1992.jukuin.keio.ac.jp) or Tomonobu Hasegawa (thaseg@keio.jp)

'Department of Pediatrics, Yokohama Municipal Citizen's Hospital, 56 Okazawa-cho, Hodogaya-ku, Yokohama, Kanagawa, Japan

${ }^{2}$ Department of Pediatrics, Keio University School of Medicine, 35 Shinanomachi, Shinjuku-ku, Tokyo, Japan

Full list of author information is available at the end of the article.
}

indicating primary hypoparathyroidism. He had a horseshoe kidney and moderate bilateral sensorineural hearing loss. Thus, he was clinically diagnosed with HDR syndrome.

We received approval for the genetic test from the institutional review board. After obtaining informed consent from his parents, we extracted genomic DNA from peripheral blood samples from the patient. We amplified all the coding exons and flanking introns of the exons in the GATA3 gene and performed direct sequencing in both directions on an autosequencer. The sequencing identified a novel heterozygous variant, c.649_653delinsAAA, p.His217Lysfs*86 in the GATA3 (NM_001002295) gene (Fig. 2). This variant was not found in either the Human Genetic Variation Database or the Exome Aggregation Consortium database.

This is the first case report of HDR syndrome with a concomitant vascular ring. The proband had a novel frameshift mutation in the GATA3 gene. This frameshift mutation leads to disruption of the zinc-finger domain and probably causes haploinsufficiency of GATA3.

The relation between GATA3 mutations and a vascular ring remains unknown. A previous study showed that, in several dog breeds, single nucleotide polymorphisms in the $T B X 1$ gene were associated with a persistent right aortic arch, a possible component of a vascular ring $[2,3]$. The $T b x 1$ gene was shown to be cooperatively regulated by GATA3 and Foxa2 proteins [4]. Another previous study using Gata3 null mice revealed that Gata3 


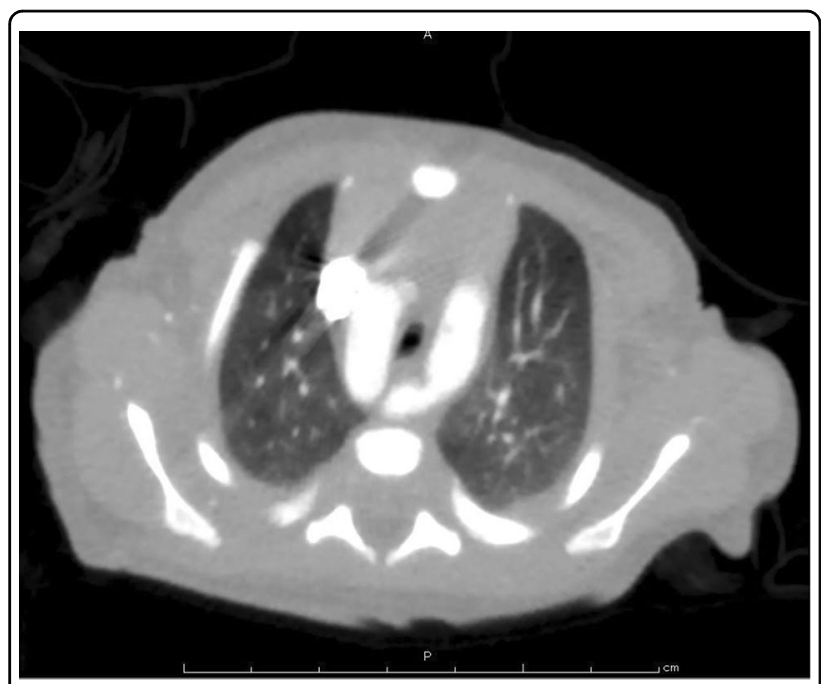

Fig. 1 A contrast computed tomography scan on the 3rd day of life. The right aortic arch, aberrant origin of the left subclavian artery, and left arterial duct formed a vascular ring.

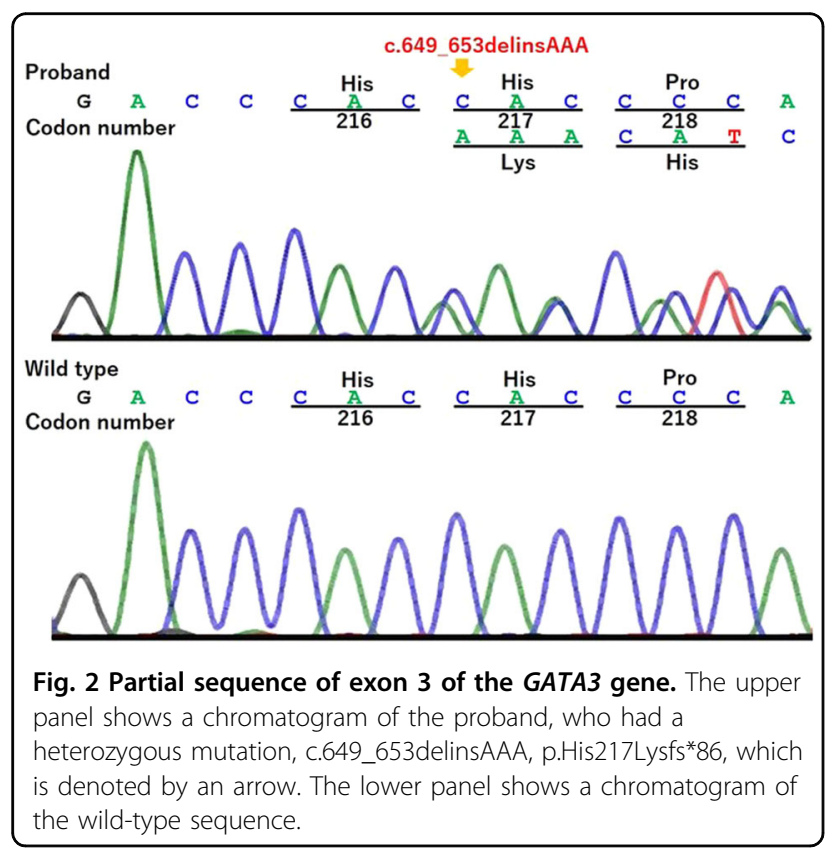

plays an important role in cardiac outflow tract formation [5]. Therefore, Gata3 mutations may contribute to the structural anomalies of the aortic arch. We speculate that a vascular ring, which is not suspected on routine echocardiography and chest radiography, remains undetected in most patients. This is a possible explanation for the lack of reports describing a vascular ring in GATA3-associated HDR syndrome. Thus, although the possibility of coincidence cannot be denied, we speculate that haploinsufficiency of GATA3 contributes to the development of a vascular ring as well as symptoms of HDR syndrome.

\section{HGV Database}

The relevant data from this Data Report are hosted at the Human Genome Variation Database at https://doi.org/10.6084/m9.figshare.hgv.2798

\section{Acknowledgements}

We thank the patient's family for participating in this study.

\section{Author details}

'Department of Pediatrics, Yokohama Municipal Citizen's Hospital, 56 Okazawa-cho, Hodogaya-ku, Yokohama, Kanagawa, Japan. ${ }^{2}$ Department of Pediatrics, Keio University School of Medicine, 35 Shinanomachi, Shinjuku-ku, Tokyo, Japan. ${ }^{3}$ Department of Neonatology, Kanagawa Children's Medical Center, 2-138-4 Mutsukawa, Minami-ku, Yokohama, Kanagawa, Japan

\section{Conflict of interest}

Tomonobu Hasegawa has the following financial relationships to disclose: Research funding from Novo Nordisk Pharma Ltd. and JCR Pharmaceuticals Co., Ltd.

\section{Publisher's note}

Springer Nature remains neutral with regard to jurisdictional claims in published maps and institutional affiliations.

Received: 24 September 2019 Revised: 19 November 2019 Accepted: 20 November 2019.

Published online: 23 December 2019

\section{References}

1. Barakat, A. J., Raygada, M. \& Rennert, O. M. Barakat syndrome revisited. Am J Med Genet. 176, 1341-8 (2018).

2. Rana, M. S., Sizarov, A., Christoffels, V. M. \& Moorman, A. F. Development of the human aortic arch system captured in an interactive three-dimensional reference model. Am J Med Genet. 164, 1372-83 (2014).

3. Philipp, U., Menzel, J. \& Distl, O. A rare form of persistent right aorta arch in linkage disequilibrium with the DiGeorge critical region on CFA26 in German Pinschers. J Hered. 102, 68-73 (2011).

4. Yamagishi, H. et al. Tbx 1 is regulated by tissue-specific forkhead proteins through a common Sonic hedgehog-responsive enhancer. Genes Dev. 17, 269-81 (2003).

5. Raid, R. et al. Lack of Gata3 results in conotruncal heart anomalies in mouse. Mech Dev. 126, 80-9 (2009). 